\title{
Attention to adjacent and separate positions in space: An electrophysiological analysis
}

\author{
HANS-JOCHEN HEINZE \\ Medical School of Hannover, Hannover, Germany \\ STEVEN J. LUCK \\ University of California, San Diego, California \\ THOMAS F. MÜNTE and ANNETTE GÖS \\ Medical School of Hannover, Hannover, Germany \\ GEORGE R. MANGUN \\ University of California, Davis, California \\ and \\ STEVEN A. HULYARD \\ University of California, San Diego, California
}

\begin{abstract}
Some theories of visuospatial attention propose that attention can be divided between separated zones of space that exclude the intervening region, whereas other theories state that the focus of attention must encompass a unitary, continuous zone. These contrasting views were evaluated in an experiment in which subjects were required to monitor two of four stimulus locations for targets; the two relevant locations were adjacent in one condition and were separated by an intervening irrelevant location in a second condition. To assess the distribution of attention across the relevant and irrelevant locations, event-related brain potentials (ERPs) were recorded to taskirrelevant "probe" stimuli that were occasionally presented at the individual stimulus locations. When the relevant locations were adjacent, probes presented at irrelevant locations elicited smaller sensory-evoked electrophysiological responses than probes presented at relevant locations, consistent with an attentional suppression of inputs from the unattended locations. When the relevant locations were separated by an irrelevant location, however, the sensory responses evoked by probes presented at this intervening irrelevant location were not suppressed, and target detection performance became slower and less accurate. These results suggest that attention forms a unitary zone that may expand to encompass multiple relevant locations but must also include the area between them; as a result, irrelevant information arising from intervening locations is not suppressed and perceptual processing is compromised.
\end{abstract}

The mechanisms by which attention is allocated to selected regions of visual space in order to optimize the intake of relevant information have been investigated extensively in recent years (for reviews see LaBerge \& Brown, 1989; van der Heijden, 1992). Most current theories propose that attentional resources can be selectively allocated to particular regions of visual space, resulting in improved processing for stimuli presented at the attended locations. Different views have emerged, however, with regard to the spatial characteristics of this attentional allocation. Shaw and colleagues (M. L. Shaw, 1978;

Correspondence should be sent to H.-J. Heinze, Medizinische Hochschule Hannover, Konstanty-Gutschow-Strasse 8, D-3000 Hannover 61, Bundesrepublik Deutschland, or S. A. Hillyard, Department of Neurosciences 0608, University of California, San Diego, 9500 Gilman Drive, La Jolla, CA 92093-0608. This research was supported by ONR Contract N00014-89-J-1806 and by grants from the DFG, NIMH (MH25594), NIH (NS-17778), and the Human Frontier Science Program.
M. L. Shaw \& P. Shaw, 1977) have proposed that visual processing capacity can be distributed flexibly over visual space to correspond with the locations where relevant stimuli are likely to occur; these attended locations may be widely dispersed and separated by unattended zones that receive fewer processing resources. A contrasting proposal is that attention is allocated in the form of a unitary focus or "spotlight" that cannot be divided among spatially noncontiguous zones (Posner, Snyder, \& Davidson, 1980). An extension of this idea is that the size of this unitary attentional focus may expand or contract in the manner of a "zoom lens" according to the size of the stimulus array to be attended (Eriksen \& St. James, 1986; Eriksen \& Yeh, 1985; Pan \& Eriksen, 1993).

In the initial formulation of Posner et al. (1980), the attentional spotlight was conceived as having a discrete boundary, producing an all-or-none enhancement of the processing of stimuli inside of the spotlight relative to stimuli outside its boundary. Recent work, however, has 
indicated that the spatial distribution of attention may take the form of a "gradient," such that the fall-off of enhanced processing in the region surrounding an attended location is gradual rather than all-or-none (Downing \& Pinker, 1985; LaBerge, 1983; LaBerge \& Brown, 1989; Mangun \& Hillyard, 1988; Shulman, Sheehy, \& Wilson, 1986; Shulman, Wilson, \& Sheehy, 1985). According to some proposals, the distribution of attention may be so broad as to involve the facilitation or inhibition of an entire visual quadrant or hemifield, and performance costs are greater if target locations cross either the vertical or horizontal meridians relative to the cued location (Hughes \& Zimba, 1987; Rizzolatti, Riggio, Dascola, \& Umiltà, 1987).

In the present study we sought to determine whether the spatial distribution of attention invariably takes the form of a single, continuous focus (spotlight or zoom lens) or whether attention may be divided into spatially noncontiguous zones. In other words, can the spotlight of attention be effectively split into separate beams when the relevant items in a display are separated by intervening irrelevant items? The experimental evidence to date on this point is mixed. M. L. Shaw (1978) found that the distribution of attention could be discontinuous across the visual field and suggested that its shape could be highly flexible. A similar conclusion was reached by LaBerge and Brown (1989). This view was supported by Egly and Homa's (1984) finding that attentional facilitation could be distributed in the form of an annulus that did not encompass the enclosed disk. Further evidence for a malleable or divisible focus of attention was provided by Beck and Ambler (1973), who found that an attention-related improvement of shape identification accuracy was the same for adjacent and separated items in a circular display. Finally, Castiello and Umiltà $(1990,1992)$ observed an inverse relationship between the size of the attended zone and reaction time (RT) for locations cued simultaneously in opposite hemifields; they concluded that spatial attention could be divided into two independent foci.

On the other hand, there is a good deal of evidence that processing is impaired when attention must be directed to spatially separated stimuli rather than to adjacent stimuli within a compact zone. This has been observed for tasks in which attention was indexed by simple RT facilitation (Posner et al., 1980), by RT for pattern identification (Eriksen \& Yeh, 1985), by RT for dot localization (Podgorny \& Shepard, 1983), and by dual-task discrimination/ detection accuracy (Hoffman \& Nelson, 1981; Sagi \& Julesz, 1986). These findings are in accord with Eriksen and Yeh's proposal that attentional resources cannot be spread across spatially separated zones without a loss of processing efficiency. In their zoom lens model, the attentional focus was considered to be unitary but adjustable in size: When the focus expands to encompass a larger area, processing resources become less concentrated and information intake is slowed.

The present study was aimed at testing certain key aspects of the zoom lens model by using both behavioral and electrophysiological measures of perceptual processing. The first aim was to determine whether the processing of form information is indeed more efficient when pairs of items to be discriminated are adjacent rather than spatially separated in a task that controls for retinal eccentricity. The design involved brief presentations of arrays of four symbols arranged in a horizontal row, centered over a fixation point (see Figure 1A). From left to right, the stimulus positions were denoted $1,2,3$, and 4. These four-item arrays were flashed in rapid sequences, and the subject was instructed to attend to two specific locations and press a button whenever the symbols at those locations matched. The primary comparison was between trial blocks in which the subject attended to a spatially compact, adjacent pair of locations in the same visual field (i.e., Locations 1 and 2, or 3 and 4) and trial blocks in which attention had to be divided between nonadjacent locations (i.e., Locations 1 and 3, or 2 and 4). According to the zoom lens model and related proposals, this pattern-matching task should be carried out more efficiently when adjacent locations are attended, resulting in faster and more accurate responses. On the other hand, if spatial attention can be divided effectively, a similar performance level would be expected in the adjacent and separated attention conditions.

The second question addressed by this study was whether attention is distributed continuously across intermediate positions when two relevant locations are spatially separated, as implied by the zoom lens model, or, alternatively, whether attention can be split into spatially separate foci that exclude the intervening positions. If, for example, Locations 1 and 3 were designated as relevant, would stimuli at Location 2 be processed as effectively as stimuli occurring at Location 3 ? This question was investigated by recording event-related brain potentials (ERPs) elicited by small "probe" stimuli that were flashed at individual stimulus positions (see Figure 1B). These probes were completely task irrelevant and served only to elicit electrophysiological responses from visual cortex that could be used as an index of perceptual processing at the individual relevant and irrelevant locations. By examining these sensory-evoked ERP responses, we sought to assess the allocation of perceptual processing resources to the relevant and irrelevant locations without requiring an overt behavioral response to stimuli presented at the irrelevant locations. Essentially, this approach uses the amplitude of the neural response elicited by a probe stimulus presented at a particular location as an index of the level of processing resources allocated to that location (see Mangun \& Hillyard, 1988, 1990a, 1991).

ERPs are scalp-recorded voltage fluctuations that reflect synchronous neuronal activity associated with sensory, motor, or cognitive events. The ERP triggered by the onset of a visual stimulus, for example, consists of a sequence of precisely timed components that arise from the progressive activation of cortical areas within the visual pathways. These components are named according to their polarity and latency (e.g. " "N1" or "N180" for the first 
major negative wave, peaking at $180 \mathrm{msec}$ poststimulus). By observing which components are affected by attentional manipulations, inferences can be made about the timing, processing level, and anatomical location of stimulus selection processes (reviewed in Hillyard \& Picton, 1987; Näätänen, 1992).

The visual ERP is particularly sensitive to the direction of spatial attention. ${ }^{1}$ In a wide variety of tasks, stimuli presented at attended locations have been found to elicit larger sensory-evoked ERP components than stimuli at unattended locations, as if attention operates as a "gain control" that modulates sensory processing within visual cortex (Mangun, Hillyard, \& Luck, 1993). Attentionrelated changes in sensory processing are first evident in the P1 component (Eason, 1981; Harter, Aine, \& Schroeder, 1982; Hillyard \& Münte, 1984; Mangun \& Hillyard, 1987, 1988, 1990b; Neville \& Lawson, 1987), which begins 70-90 msec after stimulus onset and appears to arise from lateral extrastriate visual cortex (Mangun et al., 1993; Simpson, Scherg, Ritter, \& Vaughan, 1990). Recent studies suggest that the Pl effect primarily reflects a suppression of processing at unattended locations rather than a facilitation of processing at attended locations (Luck \& Hillyard, in press; Luck et al., in press).

The P1 component has been found to index the lateralized focusing of attention to the left or right side of bilateral displays similar to those used in the present study. For example, Heinze, Luck, Mangun, and Hillyard (1990) presented rapid sequences of stimulus arrays containing two letters in each visual hemifield (see also Heinze, Mangun, \& Hillyard, 1990). Subjects were instructed to attend to the letter pairs in either the left (Positions 1-2) or right (Positions 3-4) hemifield and to press a button when the two attended letters matched each other. It was found that the P1 component elicited by bilateral letter arrays was larger over scalp sites contralateral to the attended locations than it was over ipsilateral sites, consistent with relatively greater processing within the hemisphere that received direct projections from the attended locations. In addition, task-irrelevant bar probes were occasionally presented at one or the other of the medial letter positions ( 2 and 3 ) to assess the processing of stimuli at those locations. Because the task-relevant, bilateral letter arrays elicited "compound" ERPs that reflected processing at both attended and unattended locations, the taskirrelevant probes were presented at single locations to elicit ERPs that more specifically indexed the processing resources allocated to the individual attended or unattended locations. Consistent with the hypothesis of enhanced sensory processing at the attended locations, probe stimuli presented in the attended hemifield elicited larger P1 components than probe stimuli in the unattended hemifield. Furthermore, because comparable attention-related P1 modulation effects were observed for both the taskrelevant bilateral letter arrays and the irrelevant bar probes, it was concluded that this ERP effect reflects location-based selection that is applied to all stimuli in the attended region, regardless of task relevance.
In the present study we extended this approach to assess attentional allocation under conditions in which subjects were required to detect matching stimuli at adjacent (ADJ) pairs of locations (i.e., Locations 1 and 2, or Locations 3 and 4) or separated (SEP) pairs of locations (i.e., Locations 1 and 3, or Locations 2 and 4). If the focus of attention can be effectively divided into separate zones, as implied by the models of M. L. Shaw (1978) and LaBerge and Brown (1989), we would expect larger P1 components for relevant-location probes than for irrelevantlocation probes in both the SEP and ADJ conditions. Alternatively, if dividing attention between separated locations is either impossible or inefficient, as implied by the spotlight and zoom lens models, then processing at the intervening irrelevant location would not be suppressed in the SEP condition, and the P1 elicited by probes at that location would not exhibit an amplitude reduction. More precisely, if attending to two separated locations involves the expansion of a unitary zoom lens to encompass both the relevant locations and the area between them, then the intervening irrelevant location would also fall within the focus of attention and would receive the same processing as the relevant locations. Thus, the zoom lens model would predict that the P1 elicited by probes at the inner locations ( 2 and 3 ) in the SEP condition would be about the same, whether the probe was presented at a relevant location or an irrelevant location that lay between two relevant locations. In contrast, if attention can be divided into isolated zones at the relevant locations, then probes at an irrelevant location should elicit smaller P1 amplitudes than probes at a relevant location, even if the irrelevant location lies between two relevant locations. These predictions are summarized in Figure 2.

Although the irrelevant inner location (2 or 3 ) is interposed between two relevant locations in the SEP condition, the irrelevant outer location ( 1 or 4 ) does not fall between two relevant locations in either the SEP or ADJ condition. As a result, both models of attention predict a suppression of the P1 component for irrelevant-location outer probes in the SEP condition as well as the ADJ condition (see Figure 2). Thus, the hypothesis of a single, continuous attended zone would predict similar P1 modulations for outer-location probes in both the SEP and ADJ conditions, whereas inner-location probes would exhibit attentional suppression only in the ADJ condition. In contrast, models that allow for the existence of multiple, separate attended zones would predict a P1 suppression for probes at both inner and outer irrelevant locations in both conditions.

Attention effects would also be predicted for the P1 component evoked by the task-relevant bilateral symbol arrays. In the ADJ condition, both relevant locations lie within one visual hemifield, and the P1 should therefore be larger over visual areas of the hemisphere contralateral to these locations than over the ipsilateral hemisphere. In the SEP condition, however, each hemisphere will be contralateral to one relevant location and one irrelevant location, and approximately equal P1 amplitudes should 
therefore be elicited in each hemisphere. These predictions are essentially identical for both models of attention.

\section{METHOD}

\section{Subjects}

Twelve healthy right-handed volunteers ( 4 female, 8 male) between 22 and 30 years of age participated in this experiment. All the subjects had normal or corrected-to-normal visual acuity. One subject was excluded because of poor eye movement control.

\section{Stimuli}

Stimuli were presented on a computer-controlled video monitor at a viewing distance of $70 \mathrm{~cm}$. The bilateral stimulus arrays consisted of a horizontal row of four white symbols presented on a black screen (see Figure 1). The symbols within each array were located $6.3^{\circ}$ and $11.9^{\circ}$ to the left (Positions 2 and 1) and right (Positions 3 and 4 ) of the vertical meridian, and the entire array was centered $2.9^{\circ}$ above the horizontal meridian. Each symbol's position was continuously demarcated by a dot placed $0.8^{\circ}$ above the
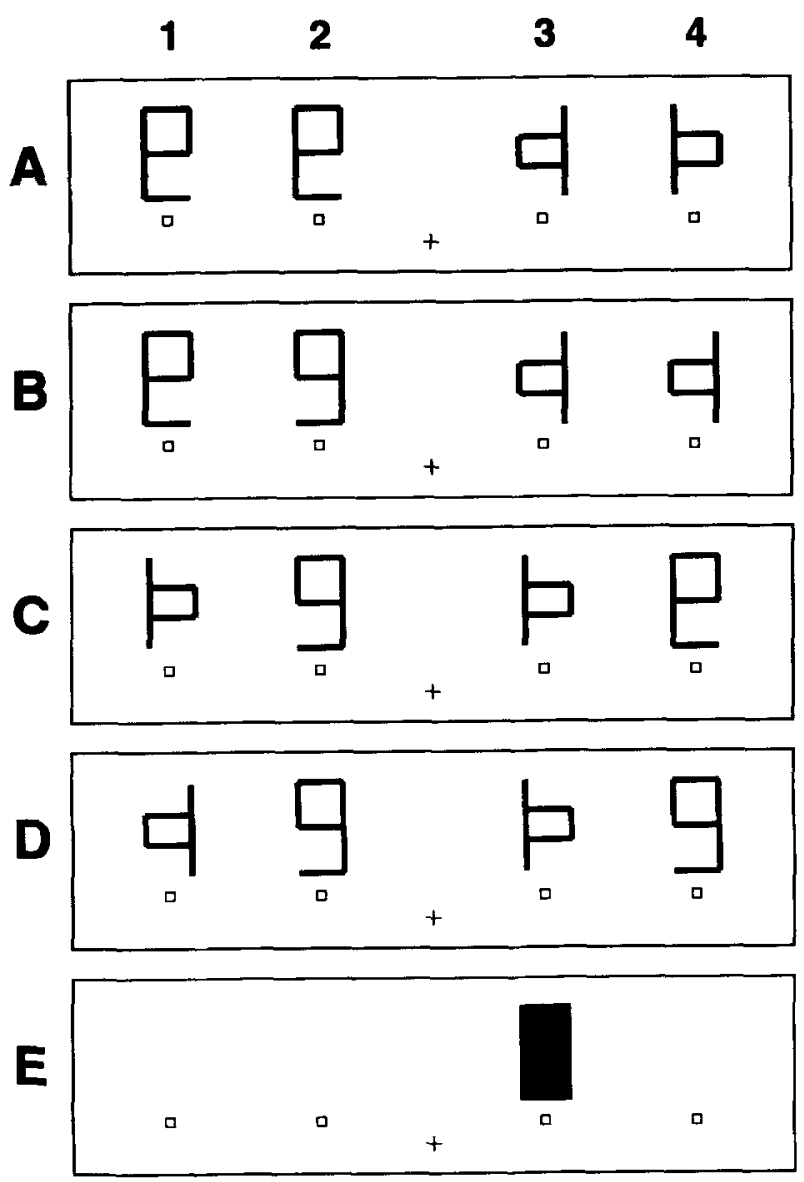

Figure 1. Examples of the stimulus arrays. Panels A-D show examples of the task-relevant bilateral stimulus arrays, with matching symbols (targets) at Positions 1 and 2,3 and 4, 1 and 3, or 2 and 4 , respectively. Panel $E$ shows a task-irrelevant probe stimulus, which could appear at any of the four locations. The task-relevant stimulus arrays and task-irrelevant probes were randomly intermixed within each trial block. The fixation cross was displayed continuously, as were the dots marking each of the four stimulus locations. horizontal meridian, and a fixation point was continuously present in the center of the display. Four different symbols were used (see Figure 1), each subtending $2.5^{\circ} \times 3.2^{\circ}$. Sixteen of the 256 possible combinations of these four symbols in the four positions were chosen for use in this experiment. Each of these combinations contained matching symbols at two of the four locations and randomly selected nonmatching symbols at the remaining two locations; each symbol array was therefore a target in one condition and a nontarget in the other three conditions (see Heinze et al., 1990, for a listing of the exact combinations). The unilateral probe stimuli consisted of white vertical bars measuring $2.5^{\circ} \times 3.2^{\circ}$, which were presented in one of the four symbol positions. Stimulus duration was $100 \mathrm{msec}$ for both the bilateral stimulus arrays and the unilateral probes.

\section{Procedure}

The subjects were seated in a sound-attenuating chamber throughout the experiment. At the beginning of each trial block, they were told to attend to Positions $1-2,3-4,1-3$, or 2-4 for that block and to press a button with the right hand when they detected matching symbols at the relevant locations. Speed and accuracy were equally stressed. The subjects were told to ignore the probe stimuli; the probes provided no task-relevant information and served only to elicit ERPs corresponding to the individual probed location.

The subjects received a total of 80 trial blocks over two sessions ( 20 blocks for each of the four conditions), run in a counterbalanced order. Each trial block consisted of a randomized sequence of 80 stimulus presentations, of which 48 were task-relevant bilateral symbol arrays (three instances of each of the 16 arrays) and 32 were task-irrelevant unilateral probes. Probe stimuli and bilateral arrays were randomly intermixed within the sequence of stimuli, with the constraint that the probes were always preceded by 1,2 , or 3 bilateral arrays. The spatial location of the probe stimulus varied unpredictably within blocks. The interval between successive stimulus onsets varied randomly between 250 and $550 \mathrm{msec}$ with a rectangular distribution, and was the same for both probes and bilateral symbol arrays. Identical symbols (targets) were present at the two relevant locations for $25 \%$ of the bilateral symbol arrays.

The following procedure was employed to ensure that the subjects did not move their eyes toward the relevant locations. At the beginning of each block, they were told to fixate the cross in the center of the display and then were told which locations were relevant for that block. Stimulus presentation then began, during which the experimenter continuously monitored the position of the eyes, which was indicated on a display of the horizontal electrooculogram (EOG). At the end of the block (approximately $30 \mathrm{sec}$ later), the subjects were again instructed to fixate. If any deviation of the eyes was detected by comparing the EOG trace during the stimulus presentation period with the pre- and postblock values, the subject was given a verbal warning and the block was discarded and repeated later in the session.

\section{Recording and Analysis}

ERPs were recorded from the scalp by using tin electrodes that were mounted in an electrode cap (Electro-Cap International) and located at standard sites spanning the scalp-F3, F4, C3, C4, P3, P4, T3, T4, T5, T6, O1, and O2 (International 10-20 System). Additional nonstandard sites were also used: $\mathrm{C} 1$, halfway between $\mathrm{Cz}$ and $\mathrm{C} 3$; $\mathrm{C5}$, halfway between $\mathrm{Cz}$ and $\mathrm{T} 3 ; \mathrm{FC1}$, halfway between $\mathrm{Cz}$ and $\mathrm{F3}$; FC5, halfway between $\mathrm{C} 3$ and $\mathrm{F} 7$; CP1, halfway between Pz and C3; CT5, halfway between C3 and T5; PO1, $0.5 \mathrm{~cm}$ lateral to the halfway point between $\mathrm{Pz}$ and $\mathrm{O} 1$; and $\mathrm{TO} 1$, halfway between $\mathrm{Ol}$ and the midpoint of a line connecting P3 and T5. Nonstandard right-hemisphere electrodes were located at the mirrorimage positions of these left-hemisphere sites. All scalp electrodes were referenced to the right mastoid. Eyeblinks were monitored with an electrode beneath the right eye, and lateral eye movements were measured as the voltage between electrodes at the left and right outer canthi (horizontal EOG). 
The electrophysiological signals were filtered with a bandpass of 0.01-70 Hz (half-amplitude cutoffs) and digitized at a rate of $250 \mathrm{~Hz}$. Automated artifact rejection was performed off line to eliminate data epochs contaminated by blinks, saccades, excessive muscle activity, and amplifier blocking. Signal-averaged waveforms were then computed for each stimulus type in each of the four conditions, by using stimulus onset as the time-locking point. Separate signal-averaged waveforms were computed for each subject for the purposes of statistical analyses, but all waveforms shown here were averaged across subjects.

The amplitude of the P1 component was quantified for each subject by measuring the mean amplitude from 90 to $150 \mathrm{msec}$ poststimulus relative to a 100 -msec prestimulus baseline. Attention effects were analyzed with repeated measures analyses of variance


Figure 2. Predicted P1 amplitudes for models in which attention must be focused in a unitary, continuous zone (UNTTARY) and for models in which attention may be divided into multiple discontinuous zones (DIVIDED). A large P1 component is predicted for probes presented within an attended zone (represented by a dotted line), and a small P1 is predicted when the probed location is outside of the attended zone(s). The predicted amplitudes are identical for both classes of models, except for the inner irrelevant location in the separate condition, which falls into an attended zone in unitary models but not in divided models. As indicated by the asterisks, P1 amplitude might be somewhat smaller in the separate condition than in the adjacent condition for the unitary models because attention is spread over a larger area; the main distinction, therefore, is that P1 amplitude should be larger at the relevant inner location than at the irrelevant inner location in the adjacent condition according to both classes of models, but should be equal at these locations in the separate condition according to unitary models. 
(ANOVAs) by using the Greenhouse-Geisser epsilon coefficient to adjust for heterogeneity of variance and covariance. Separate ANOVAs were performed for ERPs recorded at frontal electrodes (F3/4, FC1/2, FC5/6), parietal electrodes (P3/4, PO1/2), and temporal-occipital electrodes (T5/6, TO1/2, O1/2). The ADJ and SEP conditions were analyzed in separate ANOVAs. For the bilateral symbol arrays, the ANOVA factors were direction of attention (left or right), hemisphere of recording (left or right), and electrode position within a hemisphere. For the probe stimuli, separate ANOVAs were conducted for the inner (2 and 3 ) and outer (1 and 4) stimulus positions, and the factors were attention (probe at relevant or irrelevant location), probe hemifield (left or right), contralaterality of recording site (contralateral or ipsilateral to the probe hemifield), and electrode position within a hemisphere.

\section{RESULTS}

\section{Behavioral Results}

The behavioral results are shown in Table 1 . When attention was directed to adjacent positions, reaction times were 40-60 msec faster than when attention was directed to separated positions $[F(1,10)=7.70, p<.02]$. The $d^{\prime}$ measure of target detection sensitivity ranged from approximately 1.2 to 1.8 , demonstrating that this task was highly demanding. In agreement with the reaction time results, accuracy was found to be substantially reduced when the subjects attended to separated positions rather than to adjacent positions $[F(1,10)=8.65, p<.02]$. Decision bias (beta) was not significantly different in the ADJ and SEP conditions $(F<1)$.

\section{ERPs Elicited by the Unilateral Bar Probes}

ERPs elicited by bar probe stimuli at the inner and outer array positions are displayed in Figure 3, which shows waveforms recorded at electrode sites located over extrastriate visual cortex, contralateral to the visual field of the stimulus (Sites TO1 and TO2). In the ADJ condition, the P1 component was larger for relevant-location probes than for irrelevant-location probes, and similar effects were observed for probes at both the inner and outer locations (similar effects can also be observed for the P2 component). ${ }^{2}$ The P1 effect was largest at the temporaloccipital electrodes and produced a significant main effect of attention $[F(1,10)=21.03, p<.001]$ and a significant attention $\times$ electrode site interaction $[F(2,20)=$ $9.68, p<.002]$ for the inner-position probes. A significant main effect of attention was also observed for the outer-position probes in the ADJ condition $[F(1,10)=$ $6.76, p<.03]$, but the attention $\times$ electrode interaction did not approach significance. The P1 attention effect

Table 1

Mean Reaction Time, Accuracy $\left(d^{\prime}\right)$, and Standard Error of the Mean (SEM)

\begin{tabular}{|c|c|c|c|c|c|c|c|c|}
\hline & \multicolumn{4}{|c|}{ ADJ Condition } & \multicolumn{4}{|c|}{ SEP Condition } \\
\hline & \multicolumn{2}{|c|}{ Attend $1 / 2$} & \multicolumn{2}{|c|}{ Attend $3 / 4$} & \multicolumn{2}{|c|}{ Attend $1 / 3$} & \multicolumn{2}{|c|}{ Attend $2 / 4$} \\
\hline & $M$ & $S E M$ & $M$ & SEM & $M$ & $\underline{S E M}$ & $M$ & $S E M$ \\
\hline $\begin{array}{l}\text { RT (msec) } \\
d^{\prime}\end{array}$ & $\begin{array}{l}531 \\
175\end{array}$ & $\begin{array}{l}31 \\
19\end{array}$ & 543 & 38 & 591 & 32 & 584 & 33 \\
\hline & 1.75 & .19 & 1.73 & .17 & 1.20 & .17 & 1.24 & .15 \\
\hline
\end{tabular}

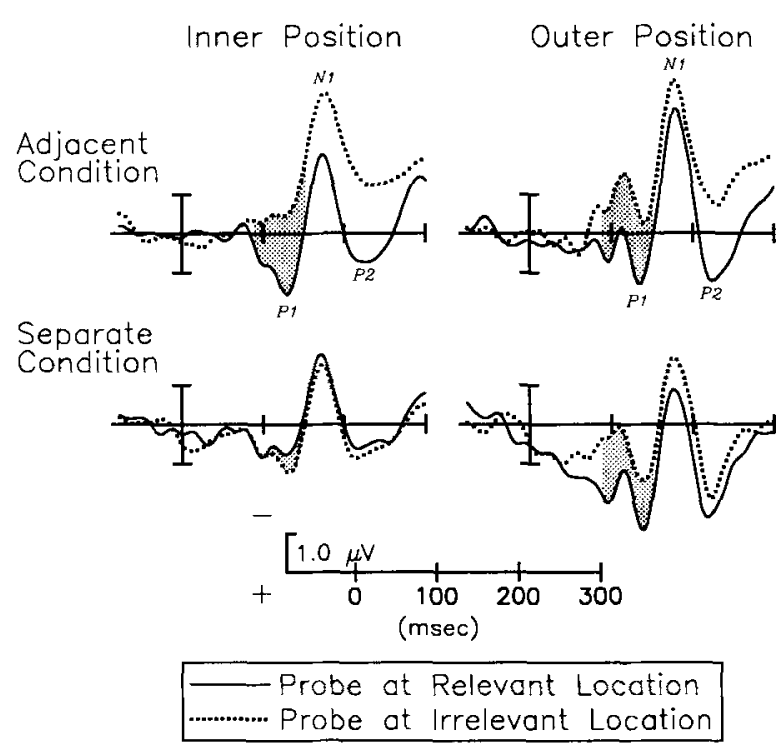

Figure 3. ERPs elicited by unilateral probe stimuli in the adjacent (top) and separate (bottom) conditions, averaged over the 11 subjects and plotted for temporal-occipital electrodes contralateral to the position of the probe stimulus. Each waveform is a plot of voltage over time, with stimulus onset at time zero; by convention, negative voltages are plotted upward. The shaded region represents the difference in amplitude for probes presented at relevant and irrelevant locations in the $\mathbf{P 1}$ measurement window (i.e., the $P 1$ attention effect). Note that large attention effects can be seen for both inner- and outer-position probe stimuli in the adjacent condition, but only for the outer-position probe stimuli in the separate condition.

tended to be larger at contralateral sites than at ipsilateral sites, but the attention $\times$ contralaterality interaction did not reach significance for either the inner $(p>.15)$ or outer $(p>.50)$ probes.

A markedly different pattern of results was observed in the SEP condition (Figure 3, lower waveforms). For inner-position probes, the P1 component was actually somewhat smaller when attention was directed to the probed location than when attention was directed to the two locations on either side of it, although this small difference was not significant $(p>.09)$. For outer-position stimuli, in contrast, the P1 component was larger when attention was directed to the probed location, especially at the temporal-occipital sites. This resulted in a significant attention $\times$ electrode site interaction for the outerposition probes $[F(2,20)=4.79, p<.02]$, although the main effect of attention did not reach significance $(p>.10)$. Thus, $P 1$ attention effects were observed for outer probes in both the ADJ and SEP conditions, but were observed for inner probes only in the ADJ condition, as predicted by the zoom lens model (see Figure 2).

Figure 4 overlays the ERP waveforms for probes presented at the inner stimulus positions and presents a summary of the P1 amplitude measurements. Compared with both relevant- and irrelevant-location probes in the SEP condition, irrelevant-location probes in the ADJ condi- 



Figure 4. ERP waveforms elicited by inner-position probe stimuli (top) and the P1 amplitude measurements from these waveforms (bottom). These data were averaged over the 11 subjects and were recorded at the contralateral temporal-occipital electrodes. The difference between relevant- and irrelevant-location P1 amplitudes was highly significant in the adjacent (ADJ) condition, but was not significant in the separate (SEP) condition.

tion produced a highly reduced $\mathrm{P} 1$ component. However, the $\mathrm{P} 1$ for relevant-location probes in the ADJ condition was only slightly enlarged relative to the SEP condition. Statistical comparisons confirmed these observations; a significant difference in P1 amplitude was observed between the SEP and ADJ conditions for irrelevant-location inner-position probes $[F(1,10)=14.44, p<.005]$, but not for relevant-location inner-position probes $(p>.10)$.

The differences between the ADJ and SEP attention effects are summarized in Figure 5, which displays the difference in amplitude between relevant- and irrelevantlocation probe $\mathrm{Pl}$ amplitudes in the form of topographic maps. In these maps, the spatial distribution of voltage has been transformed into current source density, which emphasizes superficial, cortical sources and allows more precise estimation of ERP generator locations (Pernier, Perrin, \& Bertrand, 1988). The Pl attention effects can be observed in these maps as current density foci overly- ing lateral occipital cortex, with more pronounced foci contralaterally. P1 attention effects can be observed in these maps for inner- and outer-position probe stimuli in the ADJ condition and for outer-position probe stimuli in the SEP condition, but not for inner-position probes in the SEP condition.

\section{ERPs Elicited by the Bilateral Symbol Arrays}

Figure 6 shows the ERP waveforms for bilateral stimulus arrays on target-absent trials, recorded at temporaloccipital scalp sites (TO1/2) and averaged over the 11 subjects. In the ADJ condition, the Pl component over the left hemisphere was larger when Positions 3 and 4 were relevant, and the P1 over the right hemisphere was larger when Positions 1 and 2 were relevant. The enhanced positivity over the contralateral hemisphere began approximately $80 \mathrm{msec}$ after stimulus onset and lasted 200300 msec, extending into the latency range of the P2 component. Although this attention-related positivity began in the latency range of the negative "N95" component that preceded the P1 component, it was focused over temporal-occipital scalp sites rather than the more midline scalp sites where the N95 component was maximal. This suggests that the contralateral positivity reflects a modulation of the P1 component rather than a modulation of the N95 component (see Mangun et al., 1993, for a more detailed analysis of this issue).

The contralateral positive attention effect in the ADJ condition resulted in a significant direction of attention $\times$ hemisphere interaction for the $P 1$ component $[F(1,10)=$ $23.81, p<.001]$ and was largest at the temporal-occipital electrode sites, resulting in a significant attention $\times$ hemisphere $\times$ electrode site interaction $[F(2,20)=13.88$, $p<.001]$. Similar but smaller P1 attention effects were also observed over parietal scalp sites [attention $\times$ hemisphere interaction: $F(1,10)=13.5, p<.005$ ]. No significant attention-related changes were observed at anterior scalp sites for the bilateral stimuli.

In the SEP condition (Figure 6, bottom), the temporaloccipital ERP waveforms were more positive over the left hemisphere than over the right hemisphere, but the direction of attention had no significant effect at these sites [attention $\times$ hemisphere interaction: $F(1,10)=1.1, p>$ $.30]$ or at the parietal or frontal sites $(p>.40)$.

\section{DISCUSSION}

There has been a good deal of controversy concerning the divisibility of the focus of spatial attention, and the present results provide a new source of evidence indicating that subjects cannot divide attention between two locations without also attending to the area between them. The subjects in the present experiment were found to be slower and less accurate at detecting matching stimuli at two locations when those locations were separated by an irrelevant stimulus position, as compared with when the relevant locations were adjacent. This result suggests that they employed a unitary attentional focus and were un- 


\section{Inner Position}

\section{Outer Position}


IPSI
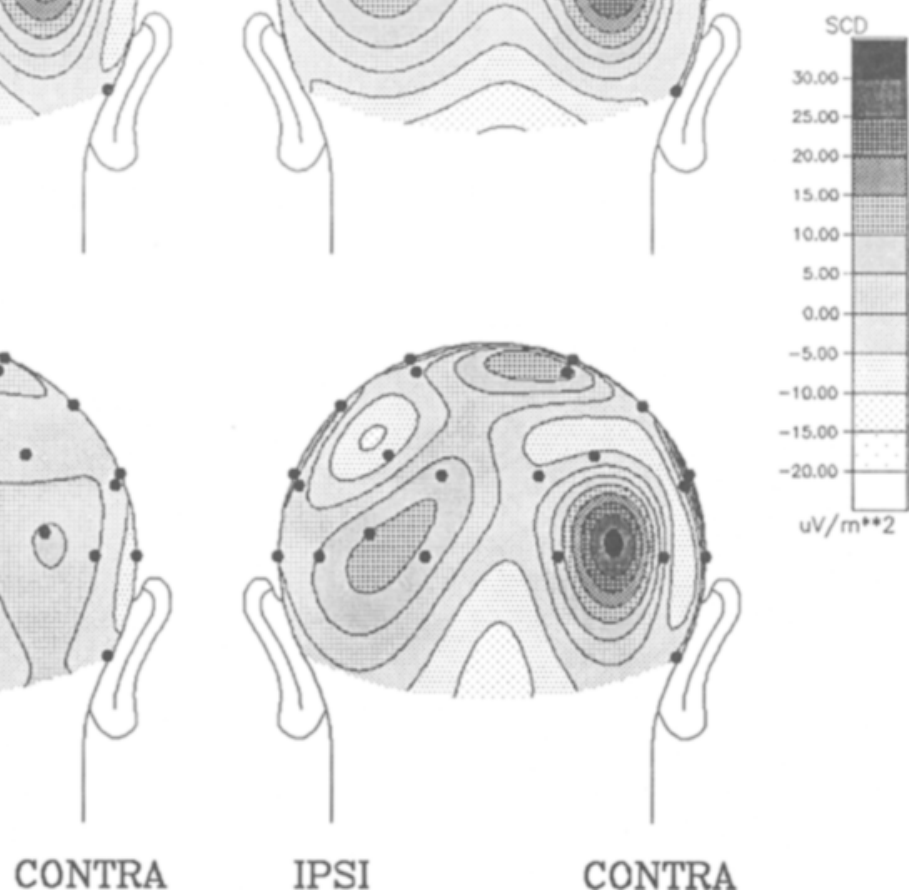

Figure 5. Isocontour maps of current source density for the $P 1$ attention effect (relevant-location probe minus irrelevant-location probe waveforms). Each level of shading represents a particular range of current density, progressing from darkly shaded positive values (current sources) to lightly shaded negative values (current sinks). The left and right sides of the schematic heads correspond to the ipsilateral and contralateral recording sites, respectively. A major contralateral source and a minor ipsilateral source can be observed at electrode sites overlying lateral extrastriate cortex. These maps were derived from the mean voltage between 80 and $120 \mathrm{msec}$, measured in difference waves in which the response to an irrelevant-location probe was subtracted from the response to a relevant-location probe. The voltage measurements were then converted into topographic maps of current source density by means of a spherical spline interpolation algorithm (Perrin, Pernier, Bertrand, \& Echallier, 1989).

able to suppress information arising from the interposed irrelevant location. This conclusion was strongly supported by the electrophysiological recordings: although the sensory-evoked P1 component was reduced for irrelevant-location probes compared with relevant-location probes under most conditions, the P1 was not suppressed when the irrelevant location was interposed between two relevant locations. Rather, the P1 amplitude for probes at the interposed irrelevant location was equivalent to the Pl amplitude observed for probes at the adjacent relevant location. Together, these results suggest that the subjects were unable to divide attention into discontinuous zones and instead employed a unitary attentional focus, even though this distribution of attention allowed information from an irrelevant interposed location to be processed extensively and thereby interfere with performance.
Although the subjects appeared to be unable to suppress sensory processing at the interposed irrelevant location, this finding does not indicate that spatial attention was completely inoperative when the two relevant locations were separated. Rather, the subjects appeared to focus attention upon a zone that included the two relevant locations and the region between them, but excluded the area that did not fall between the two relevant locations. This conclusion is based on the finding that probes at the irrelevant outer location elicited reduced P1 amplitudes compared with relevant outer-location probes, even when the two relevant locations were separated by an irrelevant inner location. This distinctive pattern of results-a P1 modulation for probes at both inner and outer locations in the ADJ condition and at outer but not inner locations in the SEP condition-strongly supports models 


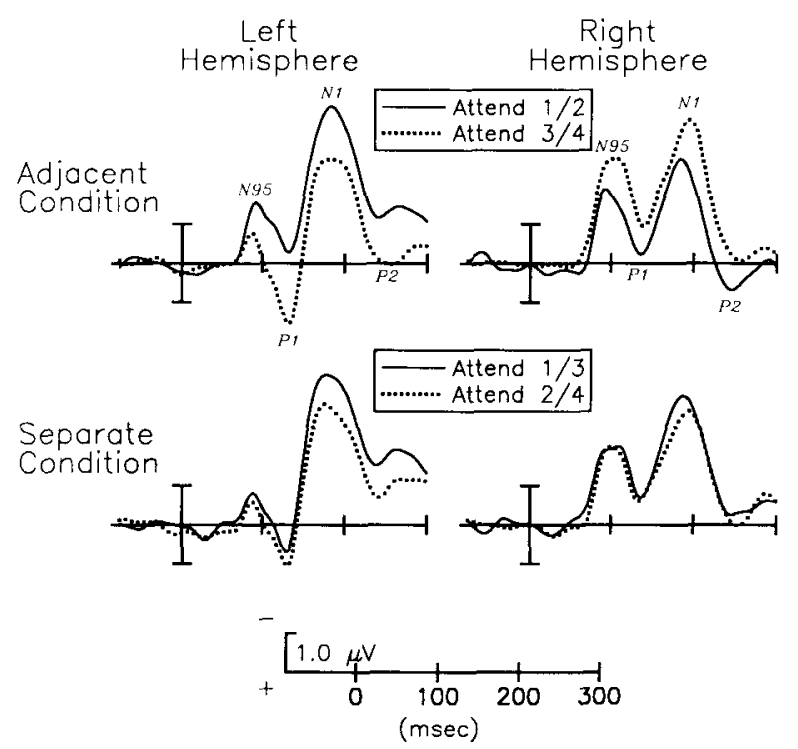

Figure 6. ERPs elicited by bilateral stimuli in the adjacent (top) and separate (bottom) conditions, averaged over the 11 subjects. These waveforms were recorded from temporal-occipital electrodes over the left and hemispheres, and exclude trials with targets (matching symbols at the relevant locations). Note the presence of a greater positivity contralateral to the relevant locations in the adjacent condition but not in the separate condition.

in which attention forms a single, continuous zone that can vary in size (see Figure 2).

The electrophysiological results suggest that task performance was slower and less accurate in the SEP condition because the subjects were unable to suppress information arising from the irrelevant location that fell between the two relevant locations in this condition. However, if attention operates in a manner analogous to a zoom lens, with an inverse relationship between resolving power and the size of the attended area (Eriksen \& St. James, 1986; Eriksen \& Yeh, 1985), then a decrement in performance would be expected in the SEP condition simply because a larger area was attended in this condition and not because of the irrelevant information that was included within this larger area. In support of this hypothesis, the P1 component elicited by relevant-location probes tended to be somewhat smaller in the SEP condition than in the ADJ condition (see Figure 4), just as would be expected if increasing the size of the attended region leads to poorer sensory processing at any given location within the region. Additional research is necessary to determine whether increases in the area encompassed by attention lead directly to reduced processing efficiency or whether the reduced efficiency is instead caused by an increase in interference from irrelevant stimuli within the expanded region, as proposed by Pan and Eriksen (1993).

In contrast with the present findings, several previous studies have shown results that are consistent with the presence of separated foci of attention (Beck \& Ambler, 1973; Egly \& Homa, 1984; M. L. Shaw \& P. Shaw,
1977). As discussed by Eriksen and Webb (1989), however, these discrepant results may be the result of a strategy in which subjects switch a unitary attentional focus back and forth between separated locations without attending to the area between them, thus simulating the simultaneous application of attention to separate regions. In other words, although subjects may be unable to divide attention into discontinuous zones at a single moment, they may effectively divide attention between locations over time. This strategy was discouraged in the present study by requiring the subjects to compare stimuli presented simultaneously at the two relevant locations. Focusing attention on one location at a given moment would be ineffective in this comparison task, because obtaining high-quality information from a single location would be insufficient for determining whether or not the characters at the two relevant locations were identical. This attribute of the experimental design may explain why evidence for a unitary attentional focus was obtained in the present experiment.

There is one way in which the current results might be compatible with a mechanism for separated attentional foci. If attention in the SEP condition takes the form of two gradients that peak at the two relevant locations, then the irrelevant inner location would fall within the tails of both gradients, which might sum together to produce an equivalent processing of stimuli at the inner irrelevant location and the surrounding relevant locations. However, given that the distance between the two relevant locations in this condition was over $18^{\circ}$, the gradients of attention would have to be extremely broad for the sum of the tails to be very large at the intervening irrelevant location. Indeed, if attentional gradients were this broad, areas of high resource allocation would almost never be separated by regions of low resource allocation, at least for detailoriented focal vision. For most practical purposes, then, an attentional system of this nature would be equivalent to models in which attention forms a single, unitary focus.

In the ADJ condition of the present experiment, both of the relevant locations were positioned within one visual hemifield, whereas the relevant locations were positioned within different hemifields in the SEP condition. Attention may operate differently for locations within, as opposed to between, hemifields (Rizzolatti et al., 1987), so it is important to consider whether the differing results obtained in the SEP and ADJ conditions could have been caused by this difference in the positioning of the relevant locations. For example, one might propose that attention could be allocated to one entire hemifield, but could not be divided between two hemifields. The present ERP results argue against this possibility, however, in that attentional modulation was observed for outer-location probes in the SEP condition (as well as in the ADJ condition), indicating that attention operated effectively at the outer locations even when divided between two hemifields. Another possibility is that attention may be divided into discontinuous zones within a hemifield but cannot be divided between hemifields, a possibility that was not 
directly tested in the present experiment. However, this would be inconsistent with previous results indicating that attention tends to spread throughout an entire hemifield but not across the vertical meridian (Hughes \& Zimba, 1987). Thus, the overall pattern of results observed in the present experiment does not seem readily explicable in terms of within- versus between-hemisphere differences in attentional allocation, although further research would be useful to clarify this point.

Although the present results provide strong evidence that a unitary focus of attention was employed under the experimental conditions used here, the generality of these findings must be considered when assessing their implications for models of attentional allocation. First, there appear to be several distinct mechanisms of visuospatial attention (see Luck \& Hillyard, in press; Posner \& Petersen, 1990), and some of these mechanisms may be be allocated more flexibly across locations than the selection process indexed by the $\mathrm{Pl}$ component. Indeed, because the subjects were able to perform the task well above chance in the SEP condition, they were presumably able to reject the information from the interposed irrelevant location to some extent, presumably at a later stage of processing. In addition, Castiello and Umiltà (1992) have proposed that attention can be divided between multiple objects but cannot be divided between separated areas of empty space, and the location markers used in the present experiment may not have been sufficiently salient to allow the subjects to maintain separate attentional foci between stimulus presentations. The nature of the perceptual task used in this study must also be considered when assessing the generality of the present results. For example, it is possible that a single attentional focus is required when subjects must perform comparisons between simultaneously presented stimuli, but that discontinuous attentional foci can be maintained when independent decisions are made at separated locations. Thus, although the comparison task was useful in the present study for discouraging the switching of attention between locations, it may represent a special case for attentional allocation strategies. Despite these limitations, however, the present findings indicate that attention cannot be allocated to discontinuous regions as flexibly as some models have suggested (LaBerge \& Brown, 1989; M. L. Shaw, 1978).

The present results are in general accordance with the zoom lens model of Eriksen and his colleagues (Eriksen \& St. James, 1986; Eriksen \& Yeh, 1985), in which the size of the attended area can be expanded or contracted depending upon the positions of the relevant stimuli. However, the results are also consistent with a moving spotlight model in which a small area is attended at any given time and the focus of attention must sweep across visual space in an analog fashion (Shulman, Remington, \& McLean, 1979; Tsal, 1983). If our subjects had switched attention back and forth between the two relevant locations in this manner, the spotlight of attention would have passed through the interposed irrelevant location in the SEP condition, thus interfering with task performance and augmenting P1 amplitude for probes presented at the inner irrelevant location. The task was designed to discourage attentional switching, however, and several other studies have indicated that attention does not move in such an analog fashion (Murphy \& Eriksen, 1987; Sagi \& Julesz, 1985); accordingly, this explanation of the present findings seems unlikely.

The present results also provide evidence concerning the level of processing at which the inferred zoom-lenslike attentional mechanism operates. In particular, three aspects of the ERP data indicate that this mechanism operates at an early stage of processing. First, the P1 attention effect began at approximately $80 \mathrm{msec}$ poststimulus, shortly after stimulus information reaches visual cortex. Second, CSD maps showed that the P1 attention effect was focused over lateral extrastriate cortex, which lies between primary visual cortex and areas specialized for pattern recognition in the inferior temporal lobe. Third, P1 attention effects were observed for both the taskrelevant bilateral symbol arrays and the task-irrelevant unilateral probe stimuli, suggesting that this attentional mechanism operates at a stage that precedes complete stimulus identification. Together, the behavioral and electrophysiological results of this experiment point to the existence of an attentional mechanism that cannot be divided between discontinuous areas and operates at a relatively early stage of processing, before stimulus identification has been completed (see also Hawkins et al., 1990; Mangun et al., 1993).

\section{REFERENCES}

BecK, J., \& AMBLER, B. (1973). The effects of concentrated and distributed attention on peripheral acuity. Perception \& Psychophysics, 14, 225-230.

Castiello, U., \& Umilti, C. (1990). Size of the attentional focus and efficiency of processing. Acta Psychologica, 73, 195-209.

Castiello, U., \& UMiltà, C. (1992). Splitting focal attention. Journal of Experimental Psychology: Human Perception \& Performance, 18, 837-848.

Downing, C. J., \& Pinker, S. (1985). The spatial structure of visual attention. In M. I. Posner \& O. S. Marin (Eds.), Attention and performance XI (pp. 171-187). Hillsdale, NJ: Erlbaum.

EASON, R. G. (1981). Visual evoked potential correlates of early neural filtering during selective attention. Bulletin of the Psychonomic Society, 18, 203-206.

EGLY, R., \& HoMA, D. (1984). Sensitization of the visual field. Journal of Experimental Psychology: Human Perception \& Performance, 10, 778-793.

ERIKSEN, C. W., \& ST. JAMES, J. D. (1986). Visual attention within and around the field of focal attention: A zoom lens model. Perception \& Psychophysics, 40, 225-240.

Eriksen, C. W., \& WebB, J. M. (1989). Shifting of attentional focus within and about a visual display. Perception \& Psychophysics, 45, 175-183.

ERIKSEN, C. W., \& YEH, Y. (1985). Allocation of attention in the visual field. Journal of Experimental Psychology: Human Perception \& Performance, 11, 583-597.

Harter, M. R., Aine, C., \& SChroeder, C. (1982). Hemispheric differences in the neural processing of stimulus location and type: Effects of selective attention on visual evoked potentials. Neuropsychologia, 20, 421-438.

Hawkins, H. L., Hillyard, S. A., Luck, S. J., Mouloua, M., DOWNING, C. J., \& WoOdWARD, D. P. (1990). Visual attention modu- 
lates signal detectability. Journal of Experimental Psychology: Human Perception \& Performance, 16, 802-811.

Heinze, H. J., Luck, S. J., Mangun, G. R., Hillyard, S. A (1990). Visual event-related potentials index focused attention within bilateral stimulus arrays: I. Evidence for early selection. Electroencephalography \& Clinical Neurophysiology, 75, 511-527.

Heinze, H. J., Mangun, G. R., \& Hillyard, S. A. (1990). Visual event-related potentials index perceptual accuracy during attention to bilateral stimuli. In C. Brunia, A. Gaillard, A. Kok, G. Mulder, \& M. Verbaten (Eds.), Psychophysiological brain research (pp. 196202). Tilburg, The Netherlands: Tilburg University Press.

Hillyard, S. A., \& MüNTE, T. F. (1984). Selective attention to color and location: An analysis with event-related brain potentials. Perception \& Psychophysics, 36, 185-198

Hillyard, S. A., \& Picton, T. W. (1987). Electrophysiology of cognition. In F. Plum (Ed.), Handbook of physiology (Vol. 5, pp. 519 584). Bethesda, MD: American Physiological Society.

Hoffman, J. E., \& Nelson, B. (1981). Spatial selectivity in visual search. Perception \& Psychophysics, 30, 283-290.

Hughes, H. C., \&imbA, L. D. (1987). Natural boundaries for the spatial spread of directed visual attention. Neuropsychologia, 25, 5-18.

LABERGE, D. (1983). Spatial extent of attention to letters and words. Journal of Experimental Psychology: Human Perception \& Performance, 9, 371-379.

LABerge, D., Brown, V. (1989). Theory of attentional operations in shape identification. Psychological Review, 96, 101-124.

Luck, S. J., Heinze, H. J., Mangun, G. R., \& Hillyard, S. A. (1990). Visual event-related potentials index focused attention within bilateral stimulus arrays: II. Functional dissociation of P1 and N1 components. Electroencephalography \& Clinical Neurophysiology, 75, 528-542.

LuCK, S. J., \& Hillyard, S. A. (in press). The role of attention in feature detection and conjunction discrimination: An electrophysiological analysis. International Journal of Neuroscience.

Luck, S. J., Hillyard, S. A., Mouloua, M., Woldorf, M. G., Clark, V. P., \& Hawkins, H. L. (in press). Effects of spatial cuing on luminance detectability: Psychophysical and electrophysiological evidence for early selection. Joumal of Experimental Psychology: Human Perception \& Performance.

Mangun, G. R., \& Hillyard, S. A. (1987). The spatial allocation of visual attention as indexed by event-related brain potentials. Human Factors, 29, 195-211.

Mangun, G. R., \& HillyaRd, S. A. (1988). Spatial gradients of visual attention: Behavioral and electrophysiological evidence. Electroencephalography \& Clinical Neurophysiology, 70, 417-428.

Mangun, G. R., \& Hillyard, S. A. (1990a). Allocation of visual attention to spatial locations: Tradeoff functions for event-related brain potentials and detection performance. Perception \& Psychophysics, 47, 532-550.

Mangun, G. R., Hillyard, S. A. (1990b). Electrophysiological studies of visual selective attention in humans. In A. B. Scheibel \& A. F. Wechsler (Eds.), Neurobiology of higher cognitive function (pp. 271-295). New York: Guilford.

MANGUN, G. R., \& HillyaRd, S. A. (1991). Modulations of sensoryevoked brain potentials indicate changes in perceptual processing during visual-spatial priming. Journal of Experimental Psychology: Human Perception \& Performance, 17, 1057-1074.

Mangun, G. R., Hillyard, S. A., \& LuCK, S. J. (1993). Electrocortical substrates of visual selective attention. In D. Meyer \& S. Kornblum (Eds.), Attention and performance XIV (pp. 219-243). Cambridge, MA: MIT Press.

MurPhy, T. D., \& ERIKSEN, C. W. (1987). Temporal changes in the distribution of attention in the visual field in response to precues. Perception \& Psychophysics, 42, 576-586.

NÄÄTÄNEN, R. (1992). Attention and brain function. Hillsdale, NJ: Erlbaum.

Neville, H. J., \& Lawson, D. (1987). Attention to central and peripheral visual space in a movement detection task: I. Normal hearing adults. Brain Research, 405, 253-267.

PAN, K., \& ERIKSEN, C. W. (1993). Attentional distribution in the visual field during same-different judgments as assessed by response competition. Perception \& Psychophysics, 53, 134-144.

Pernier, J., Perrin, F., \& Bertrand, O. (1988). Scalp current density fields: Concept and properties. Electroencephalography \& Clinical Neurophysiology, 69, 385-389.

Perrin, F., Pernier, J., Bertrand, O., Echallier, J. F. (1989). Spherical splines for scalp potential and current density mapping. Electroencephalography \& Clinical Neurophysiology, 72, 184-187.

Podgorny, P., \& SHEPARd, R. N. (1983). Distribution of attention over space. Joumal of Experimental Psychology: Human Perception \& Performance, 9, 380-393.

Posner, M. I., Petersen, S. E. (1990). The attention system of the human brain. Annual Review of Neuroscience, 13, 25-42.

Posner, M. I., SNyder, C. R. R., \& Davidson, B. J. (1980). Attention and the detection of signals. Journal of Experimental Psychology: General, 109, 160-174

Ruzzolatti, G., Riggio, L., Dascola, I., \& Umiltà, C. (1987). Reorienting attention across the horizontal and vertical meridians: Evidence in favor of a premotor theory of attention. Neuropsychologia, 25, $31-40$

SAGI, D., \& JULESZ, B. (1985). Fast noninertial shifts of attention. Spatial Vision, 1, 141-149.

SAGI, D., \& JULESZ, B. (1986). Enhanced detection in the aperture of focal attention during simple discrimination tasks. Nature, 321, 693-695.

SHAw, M. L. (1978). A capacity allocation model for reaction time. Journal of Experimental Psychology: Human Perception \& Performance, 4, 586-598.

Shaw, M. L., \& Shaw, P. (1977). Optimal allocation of cognitive resources to spatial locations. Journal of Experimental Psychology: Human Perception \& Performance, 3, 201-211.

Shulman, G. L., Remington, R. W., \& McLean, J. P. (1979). Moving attention through visual space. Journal of Experimental Psychology: Human Perception \& Performance, 5, 522-526.

Shulman, G. L., Sheehy, J. B., \& Wilson, J. (1986). Gradients of spatial attention. Acta Psychologica, 61, 167-181.

Shulman, G. L., Wilson, J., \& SheEhy, J. B. (1985). Spatial determinants of the distribution of attention. Perception \& Psychophysics, $37,59-65$.

Simpson, G. V., Scherg, M., Rutter, W., \& Vaughan, H. G., JR. (1990). Localization and temporal activity functions of brain sources generating the human visual ERP. In C. Brunia, A. Gaillard, A. Kok, G. Mulder, \& M. Verbaten (Eds.), Psychophysiological brain research (pp. 99-105). Tilburg, The Netherlands: Tilburg University Press.

TSAL, Y. (1983). Movements of attention across the visual field. Journal of Experimental Psychology: Human Perception \& Performance, 9, 523-530.

VAN DER HEIDEN, A. H. C. (1992). Selective attention in vision. London: Routledge.

\section{NOTES}

1. In all ERP recordings discussed here, the direction of gaze remained constant across different attentional conditions, and fixation was verified by recordings of the electrooculogram or related techniques.

2. In this experiment, the enhanced positivity elicited by relevantlocation probes compared with irrelevant-location probes extended over the P1 and P2 latency ranges without an amplitude enhancement of the intervening $\mathrm{N} 1$ component. As we have previously demonstrated, this pattern is typical when the stimulus sequences contain a large number of bilateral stimuli, apparently because the $\mathrm{N} 1$ attention effect is eliminated when the attended location is rapidly stimulated (see Heinze, Luck, et al., 1990; Luck, Heinze, Mangun, \& Hillyard, 1990). In the absence of the N1 attention effect, the overlap of the P1 and P2 attention effects leads to a broad positivity that extends through the latency range of the N1 component.

(Manuscript received May 5, 1993; revision accepted for publication November 12,1993 .) 